\title{
Slow Diffusive Motions in a Monolayer of Tetracosane Molecules Adsorbed on Graphite
}

\author{
H. Taub*, F. Y. Hansen ${ }^{\dagger}$, L. Criswell*, D. Fuhrmann* , K. W. Herwig ${ }^{\star}$, A. \\ Diama*, H. Mo*, R. M. Dimeo $^{\S}$, D. A. Neumann ${ }^{\S}$, and U. G. Volkmann ${ }^{\dagger \dagger}$ \\ *Department of Physics and Astronomy, University of Missouri-Columbia, Columbia, Missouri 65211, USA \\ ${ }^{7}$ Department of Chemistry, Technical University of Denmark, 207 DTU, DK-2800 Lyngby, Denmark \\ IInfineon Technologies, Memory Products, Balanstr. 73, D-81541 Munich, Germany \\ ${ }^{\ddagger}$ Spallation Neutron Source, Oak Ridge National Laboratory, 701 Scarboro Rd., Oak Ridge, TN 37830, USA \\ ${ }^{\S}$ Center for Neutron Research, National Institute of Standards and Technology, \\ Gaithersburg, Maryland 20899-8562, USA \\ ${ }^{\dagger}$ Facultad de Física, Pontificia Universidad Católica de Chile, Santiago 22, Chile
}

\begin{abstract}
Monolayers of intermediate-length alkane molecules such as tetracosane $\left(n-\mathrm{C}_{24} \mathrm{H}_{50}\right.$ or $\left.\mathrm{C} 24\right)$ serve as prototypes for studying the interfacial dynamics of more complex polymers, including bilayer lipid membranes. Using high-resolution quasielastic neutron scattering (QNS) and exfoliated graphite substrates, we have investigated the relatively slow diffusive motion in C24 monolayers on an energy/time scale of $\sim 1-36 \mu \mathrm{eV}(\sim 0.1-4 \mathrm{~ns})$. Upon heating, we first observe QNS in the crystalline phase at $\sim 160 \mathrm{~K}$. From the crystalline-to-smectic phase transition at $\sim 215 \mathrm{~K}$ to a temperature of $\sim 230 \mathrm{~K}$, we observe the QNS energy width to be dispersionless, consistent with molecular dynamics simulations showing rotational motion of the molecules about their long axis. At $260 \mathrm{~K}$, the QNS energy width begins to increase with wave vector transfer, suggesting onset of nonuniaxial rotational motion and bounded translational motion. We continue to observe QNS up to the monolayer melting temperature at $\sim 340 \mathrm{~K}$ where our simulations indicate that the only motion slow enough to be visible within our energy window results from the creation of gauche defects in the molecules.
\end{abstract}

\section{INTRODUCTION}

Over the past three decades nuclear magnetic resonance (NMR) has been used to investigate the conformational dynamics of lipid molecules in membranes [1]. In particular, measurements of the nuclear quadrupole splitting at selectively deuterated methylene groups along the alkyl chains have yielded the distribution of gauche defects along the chains in the membrane's fluid phase [2].

Recently, we have shown that the melting transition in a monolayer of intermediate-length alkane molecules $\left(n-\mathrm{C}_{\mathrm{n}} \mathrm{H}_{2 \mathrm{n}+2}\right)$, such as tetracosane $(\mathrm{n}=24)$ and dotriacontane $(\mathrm{n}=32)$, adsorbed on a graphite basal-plane surface provides an interesting analog of the gel-to-fluid transition in bilayer lipid membranes [3]. That is, our molecular dynamics (MD) simulations provide evidence of a monolayer melting transition in which intramolecular and translational order are lost simultaneously [3]. Figure 1 shows a top view of the tetracosane (C24) molecules in the MD simulation cell above and below the melting transition at $\sim 340 \mathrm{~K}$. Below the transition, we see translational order manifested by a lamellar structure. We refer to this structure as the "smectic" phase, since both the simulations and quasielastic neutron scattering measurements (see below) indicate some translational diffusive motion within the lamellae. Above the melting transition, the individual molecules have transformed to a more globular shape, i.e. have undergone a "chain-melting," and the monolayer has lost the translational order characterized by its lamellar structure. As in the case of the lipid membrane, the simultaneous "chain" melting and lattice melting of the alkane monolayer result from an abrupt increase in the number of gauche defects within the central region of each molecule.

Here we describe how high-resolution quasielastic neutron scattering (QNS) can be used to investigate the diffusive motion in a C24 monolayer occurring on a time scale of $\sim 0.1-4$ ns. We present evidence that 


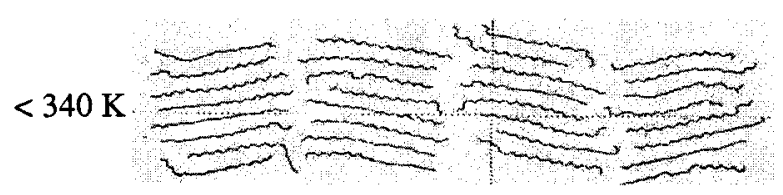

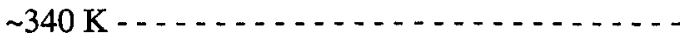

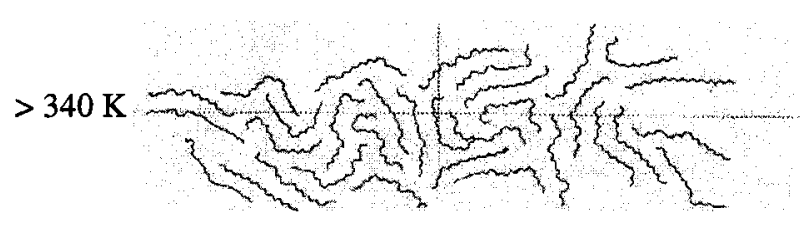

FIGURE 1. Top view showing configuration of $\mathrm{C} 24$ molecules in MD simulation cell in the "smectic" phase (upper panel) and the fluid phase (lower panel).

just below the monolayer melting temperature the principal contribution to the observed QNS is from the diffusive motions associated with conformational changes in the $\mathrm{C} 24$ molecules.

\section{EXPERIMENT}

The QNS experiments were performed on the new High Flux Backscattering Spectrometer (HFBS) at the Center for Neutron Research, National Institute of Standards and Technology [4]. The HFBS has a neutron energy resolution of $\sim 1.0 \mu \mathrm{eV}$ and a dynamic range of $0-36 \mu \mathrm{eV}$. Spectra were collected in a neutron wave vector transfer $(Q)$ range from $0.6 \AA^{-1}$ to $1.7 \AA^{-1}$. The large solid angle subtended by the silicon analyzing crystals used to select the final neutron energy makes the HFBS particularly effective for inherently small samples such as adsorbed monolayers.

The samples were prepared by depositing $\mathrm{C} 24$ from its vapor phase onto an exfoliated graphite substrate (Grafoil) in an evacuated cell at a temperature of $\sim 200$ ${ }^{\circ} \mathrm{C}$. The cells had an annular shape (to reduce multiple scattering) with a height of $30 \mathrm{~mm}$ and an inner and outer diameter of $16 \mathrm{~mm}$ and $22 \mathrm{~mm}$, respectively. Preparation details have been described elsewhere [5]. Neutron diffraction measurements at the University of Missouri Research Reactor on the same samples used for the QNS experiments were used to verify the film structure. Consistent with earlier studies [6], room temperature measurements confirmed the presence of the low-density "smectic" monolayer C24 phase shown in Fig. 1 (upper panel) and the higher-density crystalline phase, depending on coverage.

\section{RESULTS AND DISCUSSION}

We began our measurements at low temperature where we anticipated the molecular diffusive motion would be simplest to describe. Elastic neutron diffraction measurements on deuterated samples show that, upon cooling, the C24 monolayer undergoes a transition at $\sim 215 \mathrm{~K}$ from the "smectic" to a commensurate phase in which the monolayer contracts in a direction parallel to the lamellae boundaries [7]. The nearest-neighbor molecular separation in this direction becomes $4.26 \AA=\sqrt{ } 3 a_{\mathrm{g}}$ where $a_{\mathrm{g}}=2.46 \AA$ is the graphite basal-plane lattice constant compared to $\sim 4.6 \AA$ in the "smectic" phase. This higher-density crystalline phase is similar in structure to that found at room temperature when a C24 monolayer is grown from a heptane $\left(n-\mathrm{C}_{7} \mathrm{H}_{16}\right)$ solution or when a partial layer of either heptane or C24 is adsorbed above the C24 monolayer [6].

In Fig. 2, we show quasielastic spectra taken on the HFBS at two C24 coverages, $\theta=1.0$ and 1.15 layers, where unity coverage is defined as a complete monolayer of the "smectic" phase. At all temperatures in Fig. 2, both samples are in the higher-density crystalline phase described above. Due to the compression of the first layer when a partial second layer is added, we estimate that at $\theta=1.15$ there is about $7 \%$ of a second layer present. The solid line in the spectra of Fig. 2 represents the HFBS' energy resolution function. It is actually the quasielastic spectrum observed from the sample at $100 \mathrm{~K}$ where all diffusive motion within the film is frozen out.

For both coverages, we begin to observe QNS having an energy width greater than that of the HFBS' resolution function at a temperature of $\sim 160 \mathrm{~K}$. MD simulations indicate [8] that this results from motion in the crystalline phase involving a uniaxial rotation of the C24 molecules about their long axis aligned parallel to the graphite basal-plane surface (the molecules are nearly in the all-trans configuration).

Above $\sim 215 \mathrm{~K}$ at both coverages, we begin to observe QNS with sufficient intensity to determine the $Q$ dependence of its energy width. At these temperatures, the 1.0-layer sample has transformed to the "smectic" phase, while the 1.15-layer sample remains crystalline. We have fit the quasielastic intensity at each $Q$ to the sum of a Lorentzian and a delta-function convoluted with the instrumental resolution function. As shown in Fig. 3, the half-width at half-maximum (HWHM) of the Lorentzian component is essentially dispersionless at temperatures of $215 \mathrm{~K}$ and $230 \mathrm{~K}$ for both coverages. Our MD simulations indicate that in the "smectic" phase the 


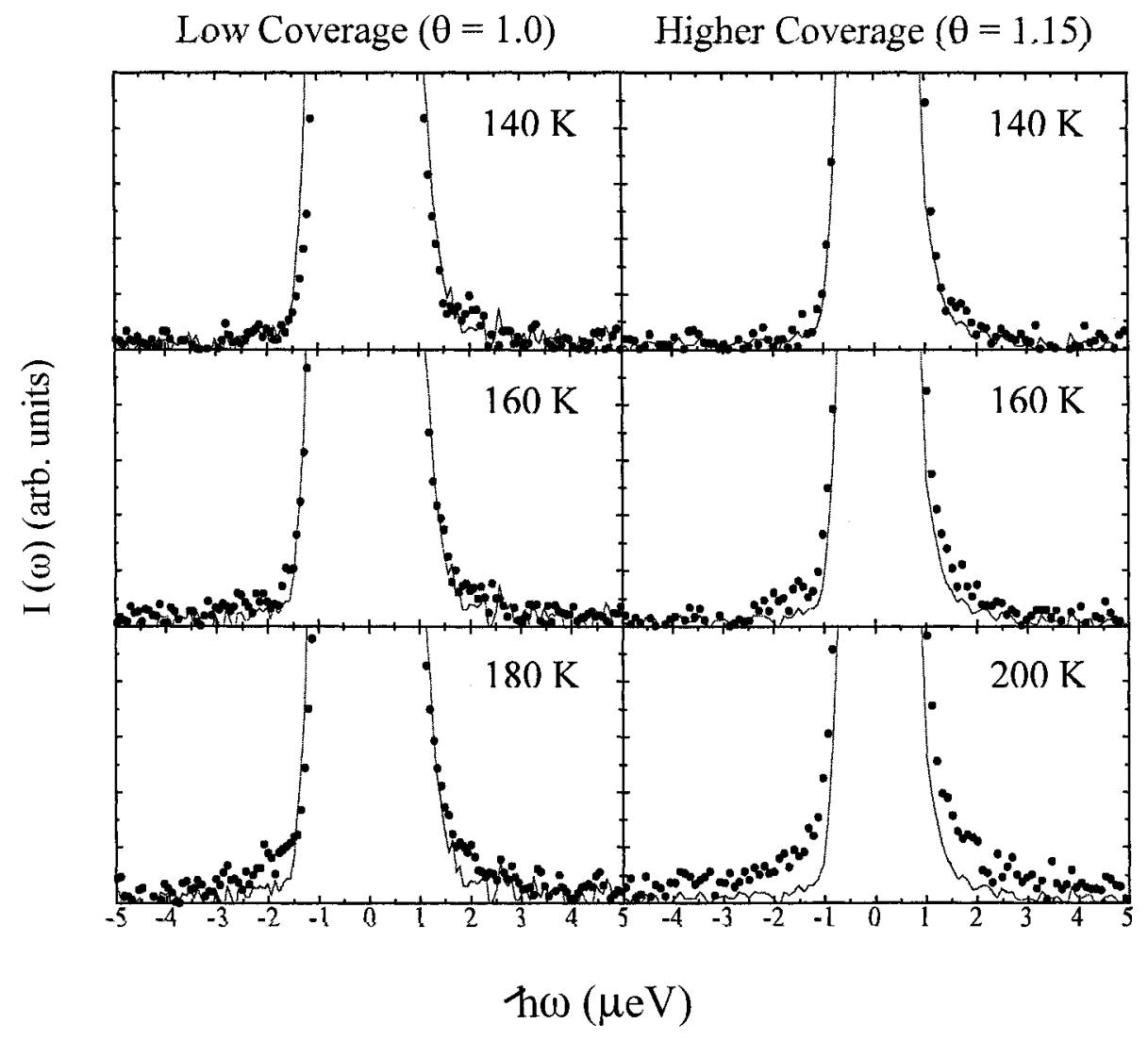

FIGURE 2. Quasielastic scattered neutron intensity measured on the HFBS from crystalline C24 monolayers at two different coverages as the temperature is increased. All QNS detected in the range $0.6-1.6 \AA^{-1}$ has been summed.

diffusive motion at $215 \mathrm{~K}$ and $230 \mathrm{~K}$ is still dominated by a uniaxial rotation of the molecules about their long axis aligned parallel to the graphite surface. Such a model is consistent with the dispersionless HWHM of the Lorentzian component of the QNS. Note that at $215 \mathrm{~K}$ and $230 \mathrm{~K}$ the 1.15 -layer sample (right panel) is still in the crystalline phase, and its HWHM's are somewhat smaller than for the 1.0-layer sample.

We see in Fig. 3 that at a temperature of $260 \mathrm{~K}$ the energy width of the QNS of the 1.0-layer sample more than triples, and the HWHM also begins to increase with $Q$. Our simulations suggest that this $Q$ dependence results primarily from nonuniaxial rotational motion and, to a lesser degree, translational diffusion.

It is interesting to compare the HWHM's for the higher-coverage sample at $260 \mathrm{~K}$. They have increased very little above their values at $230 \mathrm{~K}$, and they remain dispersionless. In fact, the magnitudes of the HWHM for the 1.15-layer sample at $260 \mathrm{~K}$ are about the same as for the 1.0-layer sample at $230 \mathrm{~K}$. These results suggest that both the nonuniaxial rotational motion and translational diffusion are inhibited in the 1.15-layer sample, which remains in the crystalline phase.

At a temperature of $330 \mathrm{~K}$, our measurements support the prediction of the MD simulations [8] that QNS should be observable due to the creation and annihilation of gauche defects in the C24 molecules. In Fig. 4, we have analyzed the $Q$ dependence of the HFBS spectra of the 1.0-layer sample at temperatures of $285 \mathrm{~K}$ and $330 \mathrm{~K}$ in the same way as for lower temperatures in Fig. 3: (a) the Lorentzian HWHM characterizing the energy width of the observed QNS; and (b) the relative intensity of the Lorentzian component in the spectra. Although the intensity becomes very weak at $330 \mathrm{~K}$, it is clear that there is still QNS within the dynamic range of the HFBS. Comparing with Fig. 3, we see that at each $Q$ the energy width of the QNS continues to increase with temperature above $260 \mathrm{~K}$. There is also a large increase in the slope of the HWHM vs. $Q$ between 260 $\mathrm{K}$ and $285 \mathrm{~K}$. For comparison, we have also plotted in Fig. 4 (dashed line) the HWHM of a Lorentzian fitted 
$\theta=1.0 \quad \theta=1.15$

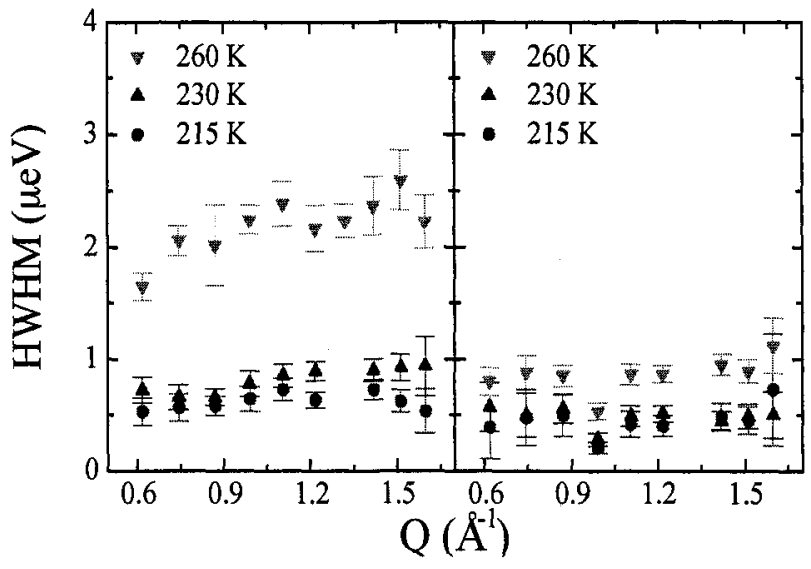

FIGURE 3. Results of fitting the quasielastic spectra at temperatures $\leq 260 \mathrm{~K}$ for the low-coverage sample (left panel) and higher coverage sample (right panel). The energy width (HWHM) of the Lorentzian component is plotted vs. wave vector transfer $Q$.

to the incoherent inelastic structure factor calculated from the MD simulation at $330 \mathrm{~K}$ after removing the center-of-mass motion from the $\mathrm{C} 24$ molecules. These calculated HWHM's characterize the contribution to the QNS from both rotational and intramolecular diffusive motions. We conclude that both their magnitude and $Q$-dependence are in qualitative agreement with experiment.

In summary, our QNS measurements and MD simulations indicate two principal temperature ranges of interest. The first of these is below $230 \mathrm{~K}$ where the diffusive motion primarily involves rotation about the long axis of the molecule aligned parallel to the surface. The second temperature range of interest is above $285 \mathrm{~K}$ where we identify the observed QNS with motions involving conformational changes of the molecules as predicted by the simulations.

\section{ACKNOWLEDGMENTS}

This work was supported by the National Science Foundation (NSF) under Grant Nos. DMR-9802476 and DMR-0109057, by the Chilean government under FONDECYT Grant No. 1010548, and by the U.S. Department of Energy through grant DE-FG0201ER45912. The neutron scattering facilities used in this work are supported in part by the NSF under Agreement No. DMR-0086210.

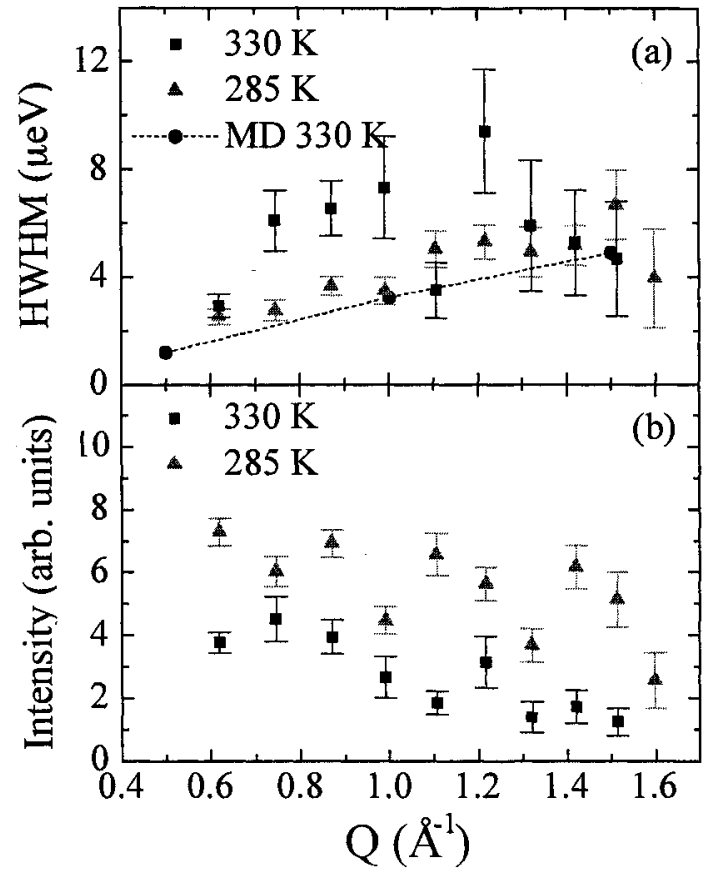

FIGURE 4. Results of fitting the quasielastic spectra at higher temperatures for the 1.0-layer sample: (a) the HWHM and (b) the intensity of the Lorentzian component vs. $Q$. In (a), the dashed line indicates values calculated from the MD simulations at $330 \mathrm{~K}$ from Ref. 8.

\section{REFERENCES}

1. See, e.g., Pastor, R.W., Venable, R.M., and Feller, S.E., Acc. Chem. Res. 35, 438-446 (2002).

2. Douliez, J.-P., Léonard, A., and Dufourc, E.J., Biophys. Jour. 68, 1727-1739 (1995).

3. Hansen, F.Y. et al., Phys. Rev. Lett. 83, 2362-2365 (1999).

4. Meyer, A. et al., Rev. Sci. Instrum. 74, 2759-2777 (2003).

5. Herwig, K.W., Matthies, B., and Taub, H., in Neutron Scattering in Materials Science, edited by D.A. Neumann, T.P. Russell, and B.J. Wuensch, Materials Research Society Symposium Proceedings 376, Pittsburgh, Pennsylvania, 1994, pp. 757-762.

6. Herwig, K.W., Matthies, B., and Taub, H., Phys. Rev. Lett. 75, 3154-3157 (1995).

7. Fuhrmann, D. et al., Surf. Sci. 482-485, 77-82 (2001); Matthies, B. et al., unpublished.

8. Hansen, F.Y., and Taub, H., this proceedings, 2003. 